Noémie Baudoin

Université catholique de Louvain (Belgique)

Sébastien Dellisse

Université catholique de Louvain (Belgique)

Dominique Lafontaine

Université de Liège (Belgique)

Liesje Coertjens

Université catholique de Louvain (Belgique)

Françoise Crépin

Université de Liège (Belgique)

Ariane Baye

Université de Liège (Belgique)

Benoit Galand

Université catholique de Louvain (Belgique)

\section{Soutien des enseignants et motivation des élèves durant la pandémie de COVID-19}

\author{
Teacher support and student motivation during the COVID-19 pandemic
}

doi: $10.18162 /$ fp.2020.688

\section{ésumé}

Cette étude vise à documenter ce qui a été mis en place par les enseignants en matière de transmission de travail scolaire et de maintien du lien social lors de la pandémie de COVID-19 en Belgique francophone ainsi quà comprendre les effets sur la motivation des élèves du secondaire ne pouvant plus se rendre à l'école. Les résultats mettent en évidence l'importance du soutien des enseignants et indiquent que les élèves recevant très fréquemment du travail à réaliser, mais n'ayant que peu de contacts avec les enseignants, se sentiraient moins capables de réaliser leur travail scolaire.

Mots-clés

Motivation, pratiques des enseignants, pandémie, enseignement à distance

Abstract

This study examines teachers' methods for assigning schoolwork and maintaining social ties during the COVID-19 pandemic in Frenchspeaking Belgium. It also investigates the effects on motivation in secondary-school students who could not attend school in person. The results highlight the importance of supporting teachers and indicate that students who are frequently assigned schoolwork but have few contacts with teachers feel less capable of accomplishing their

work.

\section{Keywords}

Motivation, teaching practices, pandemic, remote learning

\section{Introduction}

La crise due à la COVID-19 a conduità la fermeture desétablissements scolaires dans de nombreux pays. Selon l'UNESCO, plus de $60 \%$ des élèves dans le monde ont été affectés par la fermeture prolongée de leur école ou par des limitations extrêmes des conditions habituelles d'apprentissage. En quelques jours, de nombreux élèves sont notamment passés d'un enseignement classique en présentiel à un enseignement à distance, bousculant leurs habitudes ainsi que celles de leurs enseignants. À la suite de cette situation, on peut s'attendre, selon un rapport de la Commission européenne (Di Pietro, Biagi, Costa, Karpiński et Mazza, 2020), à des répercussions multiples sur la motivation, les apprentissages ou le bien-être des élèves. Concernant la motivation plus spécifiquement, elle serait selon ce rapport affectée négativement par l'absence des pairs qui contribuent habituellement à susciter la motivation en classe. Toujours selon ce rapport, il est également probable que la suspension du redoublement ou des évaluations dans certains pays affecte la motivation extrinsèque des élèves. Habitués à travailler pour des points ou par peur de recommencer leur année, certains pourraient en effet être moins motivés par les activités d'apprentissage. Pourtant, la nécessité d'une continuité des apprentissages durant la période de pandémie fait aujourd'hui consensus. Impliquer les élèves dans leurs apprentissages dans ce contexte particulier constitue donc un enjeu essentiel encore peu documenté. L'objectif de cet article est d'étudier cette question et d'outiller ainsi les acteurs de l'éducation soucieux de mettre en œuvre des pratiques soutenant la motivation des élèves en contexte de pandémie. 


\section{Motivation des élèves}

La motivation en contexte scolaire est le processus par lequel un élève s'investit dans les activités scolaires. Un élève est considéré comme motivé lorsque l'on peut observer différents indicateurs de cette dynamique motivationnelle (Fredricks, Blumenfeld et Paris, 2004) : des comportements dénotant son implication dans l'activité (engagement comportemental), la mise en œuvre de stratégies cognitives et métacognitives adaptées (engagement cognitif) et des réactions affectives positives en situation d'apprentissage (engagement émotionnel). Dans une perspective de dynamique motivationnelle, l'engagement de l'élève est conçu comme étant influencé par les représentations que celui-ci se fait concernant, d'une part, sa capacité à réussir la tâche et, d'autre part, la valeur qu'il attribue à cette tâche (Eccles et Wigfield, 1995). La valeur attribuée à la tâche peut notamment varier selon l'intérêt, l'importance ou l'utilité accordé par l'élève à l'activité en question. Est-il intrinsèquement intéressé par ce qu'on lui propose? Est-ce important pour lui, pour son identité, de réaliser cette tâche ? Et, enfin, est-ce utile dans son quotidien ou pour plus tard ? Ces différentes représentations seraient elles-mêmes affectées à la fois par des caractéristiques propres à lélève et des caractéristiques de son environnement. Selon l'approche de Bandura (1980) concernant le sentiment d'efficacité personnelle, les croyances relatives à sa capacité à réussir une tâche seraient notamment influencées par les réussites antérieures de lélève, son état affectif, le fait de recevoir un retour et des encouragements, mais aussi d'observer d'autres élèves réaliser et réussir la tâche. Le modèle théorique de l'autodétermination (Deci et Ryan, 2000) soutient quant à lui que les pratiques soutenant les besoins d'affiliation, de compétence et d'autonomie des élèves seraient bénéfiques à la motivation intrinsèque ou à l'intérêt des élèves. Ces pratiques permettraient également d'internaliser, de faire siennes, les raisons socialement approuvées de s'investir dans les activités scolaires, conduisant les élèves à accorder davantage d'utilité et d'importance à celles-ci. Les effets bénéfiques du soutien des enseignants sur la motivation des élèves sont aujourd'hui amplement documentés dans le contexte classique de cours donnés en classe (Federici et Skaalvik, 2014). À la lumière de ces différents cadres théoriques, soutenir la motivation des élèves en contexte de pandémie constitue indéniablement un défi de taille pour les enseignants.

\section{Contexte de l'enseignement en Belgique francophone pendant la pandémie de COVID-19}

En Belgique francophone, les cours ont été suspendus à partir du lundi 16 mars 2020 et un confinement a été imposé à l'ensemble de la population à partir du mercredi 18 mars. Les écoles ont reçu comme message que les cours ne pouvaient plus être dispensés, mais que des travaux à domicile pouvaient être prévus. Dans un souci d'égalité d'accès aux apprentissages, ces travaux ne pouvaient porter que sur de la matière préalablement abordée en classe et ne pouvaient pas faire l'objet d'une évaluation sommative. Les enseignants étaient invités à être attentifs au caractère proportionné des travaux transmis et à faire en sorte que chaque élève puisse les réaliser en autonomie. Ceux-ci devaient également s'assurer que tous les élèves du groupe-classe puissent effectivement accéder à ce qui leur était proposé (supports papier, enseignement en ligne, etc.). Le maintien d'un lien social via les moyens technologiques disponibles était également fortement recommandé. À l'exception de ces quelques balises, une grande marge de manœuvre était laissée aux équipes éducatives concernant les modalités de mise en œuvre de ces travaux à domicile et le maintien du lien social. 
À la suite du déconfinement entamé début mai, une reprise progressive pour certains groupes d'élèves prioritaires (années certifiantes ou orientantes, début de scolarité primaire...) a eu lieu à partir du 18 mai. Cette reprise n'a cependant été que partielle, tout particulièrement pour les élèves du secondaire, où une majorité d'élèves n'a pas été concernée.

$\mathrm{Vu}$ ce contexte, l'enjeu de la motivation des élèves apparaît particulièrement sensible en Belgique francophone. En effet, outre les aspects abordés dans le rapport de l'Union européenne (Di Pietro et al., 2020), le fait de n'aborder que de la matière préalablement vue en classe pourrait conduire les élèves à juger le travail qui leur est proposé comme moins intéressant et utile. La Belgique francophone apparaît également comme un espace d'investigation intéressant au vu de la liberté laissée aux établissements et aux enseignants. Cette liberté a probablement eu comme conséquence une grande diversité de pratiques et de situations, permettant d'examiner d'éventuels effets différenciés sur la motivation des élèves.

\section{Questions de recherche}

La présente étude vise tout d'abord à documenter ce qui a été réalisé en matière de transmission de travail scolaire et de maintien du lien social en Belgique francophone durant la pandémie de COVID19, plus particulièrement auprès des élèves de l'enseignement secondaire. À quelle fréquence du travail scolaire a-t-il été transmis ? Les élèves ont-ils été régulièrement en contact avec leurs enseignants ? Et ont-ils eu la possibilité d'interagir de vive voix avec ceux-ci ?

Dans un deuxième temps, cette étude cherche à comprendre comment la manière dont la continuité du travail scolaire a été mise en œuvre peut affecter la motivation des élèves en contexte de pandémie. En s'appuyant sur les cadres théoriques existants, il est apparu pertinent d'examiner dans ce contexte l'effet du soutien de l'enseignant sur la motivation des élèves, en plus de celui de la fréquence du travail transmis, des contacts avec les enseignants et des interactions de vive voix avec ceux-ci. La motivation est ici appréhendée comme un processus dynamique en prenant en considération à la fois la valeur accordée par l'élève à la tâche, son sentiment d'efficacité personnelle par rapport aux activités proposées ainsi que son engagement comportemental vis-à-vis du travail scolaire.

Enfin, de manière exploratoire, les commentaires libres récoltés dans le cadre de l'enquête seront exploités pour discuter et enrichir nos résultats afin de mieux comprendre comment soutenir la dynamique motivationnelle des élèves dans le contexte spécifique d'une pandémie.

\section{Méthodologie}

\section{Procédure}

Cette étude est réalisée à partir des données d'une enquête plus large concernant le bien-être et la motivation des élèves du secondaire en Belgique francophone menée durant le mois de juin 2020. Cette enquête a été financée et réalisée en collaboration avec l'administration générale de l'enseignement de la Fédération Wallonie-Bruxelles. Elle a été largement diffusée en ligne via différents canaux. L'administration générale de l'enseignement a notamment diffusé un communiqué de presse, qui a fait l’objet d'une dépêche BELGA reprise sur plusieurs sites d'actualité belge. Des courriers électroniques 
officiels ont été envoyés à différents acteurs de l'éducation (directions d'école, associations de parents, ...). De leur côté, les chercheurs ont également diffusé l'enquête via les canaux de diffusion interne à leurs universités respectives.

Le message diffusé présentait les objectifs de l'enquête aux parents et résumait les règles en matière de protection des données privées et d'éthique. Si ceux-ci donnaient leur consentement, ils étaient invités à transmettre le questionnaire à leur enfant via le lien URL ou le code QR. Ce questionnaire, hébergé sur la plateforme Qualtrix, était accessible via un ordinateur ou un smartphone. Les jeunes devaient également donner leur consentement actif avant d'accéder au questionnaire auto-rapporté composé de 26 questions à choix multiples et finalement d'une question ouverte. Celle-ci permettait de laisser un éventuel commentaire à la question «As-tu envie d'ajouter quelque chose par rapport au confinement ou par rapport à la manière dont tu te sens en ce moment ? ». La durée moyenne estimée pour compléter l'enquête était de 15 minutes.

\section{Échantillon}

Parmi les 6733 répondants qui ont accepté de répondre à l'enquête durant le mois de juin, 498 ont répondu à moins de $20 \%$ du questionnaire et ont donc été écartés de l'échantillon. Sur ces 6235 questionnaires valides, nous avons conservé ceux des élèves affirmant être dans l'enseignement secondaire (plutôt qu'en primaire, dans l'enseignement supérieur, etc.) et âgés de 11 à 24 ans. L'échantillon est donc finalement constitué de 6015 répondants. L'échantillon comporte davantage de filles $(59,5 \%)$ et se répartit plutôt équitablement entre les différentes années scolaires $(14,8 \%$ en première, $14 \%$ en deuxième, $16,8 \%$ en troisième, $17,8 \%$ en quatrième, $20 \%$ en cinquième et $17 \%$ en sixième, y compris les élèves de septième de la filière professionnelle). Parmi les répondants, 64,7 \% rapportent ne jamais avoir redoublé, $15,6 \%$ rapportent avoir redoublé une fois et 7,5\% avoir redoublé deux fois ou plus $(12,2 \%$ se sont abstenus). Ces taux de redoublement sont assez nettement inférieurs à ceux habituellement observés en Fédération Wallonie Bruxelles et laissent supposer que notre échantillon n'est pas représentatif de l'ensemble des élèves qui y sont scolarisés. D'autres indicateurs (filière fréquentée, nombre de livres à la maison) donnent à penser que l'échantillon est plus favorisé socialement que la population de référence.

Dans le cadre de cette étude, nous allons nous focaliser sur les élèves de secondaire dont les cours à l'école sont suspendus. Les analyses seront donc réalisées sur les données récoltées entre le 4 et le 18 juin auprès des élèves des années pas (ou peu) concernées par la reprise partielle des cours et qui ont mentionné que les cours n'avaient pas repris pour eux en présentiel. L'échantillon pour la partie quantitative se compose donc de 3009 élèves, dont 22,2 \% sont en première secondaire, $24,2 \%$ en troisième, $25,1 \%$ en quatrième et $28,5 \%$ en cinquième. Les répondants ont en moyenne 15 ans et la majorité sont des filles (59,8\%). Notons également que, à la suite des analyses descriptives, il est apparu que 52 répondants indiquent ne jamais avoir reçu de travail à domicile. Étudier la motivation à réaliser des travaux quand il n'y en a pas eu n'ayant pas de sens, ces individus ont donc été écartés pour les analyses de régression.

Lorsque nous nous pencherons sur les commentaires libres laissés à la fin du questionnaire par certains répondants, nous conserverons toutefois l'ensemble de l'échantillon, estimant que les élèves de retour à l'école peuvent potentiellement aussi émettre un avis concernant ce qui s'est passé quelques semaines plus tôt. Parmi les 6015 répondants, 1717 (28,5 \%) ont laissé un commentaire.

4 - Formation et profession 28(4 hors-série), 2020 


\section{Mesures}

\section{Motivation}

La valeur de la tâche attribuée par les élèves au travail à domicile était mesurée au travers de trois dimensions : l'intérêt, l'utilité et l'importance. Pour chaque dimension, deux items ont été adaptés d'une échelle existante (voir Olivier, Galand, Hospel et Dellisse, 2020) ; les élèves étaient invités à répondre sur une échelle à 5 points allant de " pas du tout d'accord » à « tout à fait d'accord » avec un point neutre. L'intérêt était notamment mesuré par «Les activités proposées me font découvrir des choses très intéressantes ", l'utilité par "Ce travail ne sert à rien $(R)$ » et enfin l'importance par "C'est important pour moi de bien faire le travail qui m’est demandé ». L'alpha de Cronbach pour cette échelle de valeur comprenant 6 items est de .82 .

Le sentiment d'efficacité personnelle relatif au travail à réaliser à domicile était mesuré par 4 items tirés d'une échelle existante (Galand et Philippot, 2002) et adaptés à la situation particulière dont, par exemple, « je suis capable de réaliser facilement ce que l'on me demande pour l'école » ou « je suis certain'e que je peux comprendre ce que l'on apprend pour le moment ». Les élèves étaient invités à se situer par rapport à ces items sur une échelle à 5 points allant de « pas du tout d'accord » à « tout à fait d'accord ». L'alpha de Cronbach est de .78.

L'engagement comportemental est mesuré par 6 items abordant la participation, le respect des consignes ou la passivité des élèves par rapport au travail à domicile. Quelques exemples d'items sont " J'essaie vraiment de répondre lorsqu'une question est posée ou une activité proposée ", "Je m'applique pour faire mon travail » ou encore "Je n'essaie même pas de le faire $(R)$ ». Ces items sont adaptés d'échelles existantes (voir Hospel, Galand et Janosz, 2016) et les élèves étaient invités à répondre sur une échelle à 5 points allant de « jamais » à « très souvent ». L'alpha de Cronbach est de .82 .

\section{Continuité du travail scolaire et du lien social}

Les questions relatives à la fréquence de la transmission de travail à domicile, des contacts avec les enseignants et des interactions de vive voix avec ceux-ci ont été créées spécifiquement pour cette enquête. Les élèves étaient invités à répondre sur une échelle à 5 points ("Jamais »; "Moins d'une fois par semaine »; «Environ une fois par semaine »; «2-3 fois par semaine »; "Tous les jours ou presque ») aux items suivants : "Dans la situation actuelle, je reçois du travail scolaire à faire à la maison »; «Dans la situation actuelle, j'ai des contacts avec un·e ou des enseignant·e·s (cours, échanges de messages, de vidéos...) "; "Dans la situation actuelle, je discute « de vive voix » avec un·e ou des enseignant·e·s (à l'école, au téléphone, par appel vidéo...)».

\section{Perception de soutien de la part des enseignants}

Le soutien perçu de la part des enseignants est mesuré par 6 items adaptés d'une échelle existante (Galand et Hospel, 2011). Le soutien perçu de la part des enseignants concernait tant un soutien émotionnel qu'un soutien instrumental orienté vers le soutien des apprentissages (voir Federici et Skaalvik, 2014). Les élèves étaient invités à répondre sur une échelle à 5 points allant de « jamais » à " très souvent ». Les items de cette échelle sont présentés dans le tableau 2 et l'alpha de Cronbach est de .86 . 


\section{Variables de contrôle}

Les élèves avaient la possibilité de préciser s'ils étaient une Fille (codé 0), un Garçon (codé 1) ou s'ils se considéraient comme Autre. Afin de faciliter les analyses et leur interprétation, les rares élèves ayant coché «Autre » ont été codés comme valeur manquante dans le cadre de cette étude. Les élèves étaient également invités à rapporter s'ils n'avaient jamais redoublé (0), s'ils avaient déjà redoublé une fois (1) ou s'ils avaient déjà redoublé deux fois ou plus (2). Enfin, le niveau socio-économique (SES) de l'élève était appréhendé par le nombre de livres à la maison. Les élèves étaient invités à répondre à la question "Combien de livres y a-t-il chez toi ? (Ne compte pas les magazines ni les manuels scolaires) » selon les modalités " Pas de livre ou très peu de livres (de 0 à 10 livres) " (codé 1 ), " Assez de livres pour remplir une étagère (de 11 à 25 livres) » (codé 2), «Assez de livres pour remplir une bibliothèque (de 26 à 100 livres) » (codé 3), «Assez de livres pour remplir deux bibliothèques (de 101 à 200 livres) » (codé 4) et « Assez de livres pour remplir trois ou plus de trois bibliothèques (plus de 200 livres) » (codé 5).

\section{Résultats}

\section{Analyses descriptives}

Le premier objectif de cette étude est de documenter comment la continuité du travail scolaire et le maintien du lien social ont été mis en œuvre par les enseignants en Belgique francophone. Nos données indiquent qu'une majorité d'élèves a reçu du travail scolaire à réaliser (70,9\%). Une courte majorité rapporte avoir été régulièrement en contact avec les enseignants (54,5\%). Enfin, une minorité d'élèves ont eu l'occasion d'interagir oralement avec leurs enseignants environ une fois par semaine ou davantage $(27,5 \%)$. Les fréquences précises sont présentées dans le tableau 1.

\section{Tableau 1}

Fréquence des réponses concernant la continuité du travail scolaire et du lien social

\begin{tabular}{llllll}
\hline Dans la situation actuelle, & Jamais & $\begin{array}{l}\text { Moins d'une } \\
\text { fois par } \\
\text { semaine }\end{array}$ & $\begin{array}{l}\text { Environ } \\
\text { une fois par } \\
\text { semaine }\end{array}$ & $\begin{array}{l}\mathbf{2 - 3} \text { fois par } \\
\text { semaine }\end{array}$ & $\begin{array}{l}\text { Tous les } \\
\text { jours ou } \\
\text { presque }\end{array}$ \\
\hline $\begin{array}{l}\text { je reçois du travail scolaire } \\
\text { à faire à la maison }\end{array}$ & $1,8 \%$ & $8,5 \%$ & $18,9 \%$ & $38,4 \%$ & $32,5 \%$ \\
\hline $\begin{array}{l}\text { j'ai des contacts avec un.e } \\
\text { ou des enseignant·e.s }\end{array}$ & $5,9 \%$ & $14,3 \%$ & $25,3 \%$ & $34 \%$ & $20,5 \%$ \\
\hline $\begin{array}{l}\text { je discute « de vive voix» } \\
\text { avec un.e ou des enseignant.e.s }\end{array}$ & $53,5 \%$ & $19 \%$ & $17,7 \%$ & $8,3 \%$ & $1,5 \%$ \\
\hline
\end{tabular}

Concernant la perception des pratiques de soutien de la part des enseignants (voir tableau 2), une majorité d'élèves rapportent que les enseignants n'hésitent (très) souvent pas à réexpliquer quelque chose si on le demande $(67,7 \%)$, donnent (très) souvent des consignes claires qui aident à savoir ce qu'il faut faire $(58 \%)$ et ne laissent jamais ou rarement les élèves se débrouiller seuls avec leurs difficultés 
(58,1\%). Toutefois, seule une minorité rapporte que les enseignants s'adaptent (très) souvent aux difficultés que les élèves rencontrent (46,3\%), s'intéressent (très) souvent à ce qu'ils vivent $(33,6 \%)$ ou prennent (très) souvent le temps d'échanger avec les élèves à propos de la situation particulière vécue $(28,8 \%)$.

\section{Tableau 2}

Fréquence des réponses concernant la perception de soutien de la part des enseignants

\begin{tabular}{l|l|l|l|l|l}
\hline $\begin{array}{l}\text { Actuellement, } \\
\text { mes enseignants ... }\end{array}$ & Jamais & Rarement & Parfois & Souvent & Très souvent \\
\hline $\begin{array}{l}\text { s'intéressent vraiment à ce } \\
\text { que les élèves vivent. }\end{array}$ & $10 \%$ & $22,8 \%$ & $33,6 \%$ & $24,4 \%$ & $9,2 \%$ \\
\hline $\begin{array}{l}\text { laissent les élèves se débrouiller } \\
\text { seuls avec leurs difficultés. }\end{array}$ & $25,7 \%$ & $32,4 \%$ & $23 \%$ & $12,5 \%$ & $6,3 \%$ \\
\hline $\begin{array}{l}\text { prennent le temps d'échanger avec } \\
\text { les élèves à propos de la situation } \\
\text { particulière que nous vivons. }\end{array}$ & $14,6 \%$ & $25,1 \%$ & $31,5 \%$ & $19,9 \%$ & $8,9 \%$ \\
\hline $\begin{array}{l}\text { n'hésitent pas à réexpliquer } \\
\text { quelque chose si on le demande. }\end{array}$ & $4,5 \%$ & $9,4 \%$ & $18,5 \%$ & $37,7 \%$ & $30 \%$ \\
\hline $\begin{array}{l}\text { donnent des consignes claires qui } \\
\text { nous aident à savoir ce qu'il faut } \\
\text { faire. }\end{array}$ & $3,6 \%$ & $11,7 \%$ & $26,7 \%$ & $41,1 \%$ & $16,9 \%$ \\
\hline $\begin{array}{l}\text { s'adaptent aux difficultés que les } \\
\text { élèves rencontrent. }\end{array}$ & $7,8 \%$ & $16,9 \%$ & $29 \%$ & $30 \%$ & $16,3 \%$ \\
\hline
\end{tabular}

\section{Analyses multivariées}

Afin d'examiner comment la continuité du travail scolaire et du lien social a affecté la motivation des élèves, des régressions multiples ont été réalisées au moyen du logiciel SPSS. Différents modèles de régression ont été successivement testés en introduisant l'un après l'autre des blocs de variables constitués tout d'abord des variables de contrôle, ensuite des variables liées à la fréquence de transmission du travail à domicile, des contacts avec les enseignants et des interactions de vive voix avec ces derniers, et finalement du soutien perçu de la part des enseignants. Ces mêmes modèles successifs ont été testés pour les trois variables dépendantes liées à la dynamique motivationnelle en contexte de pandémie : la valeur perçue, le sentiment d'efficacité personnelle et l'engagement comportemental. Dans un souci de parcimonie, seules les variables significatives seront prises en compte dans les modèles finaux (voir tableau 3).

En ce qui concerne la valeur perçue du travail à domicile, nos analyses indiquent une relation positive avec la fréquence des contacts avec les enseignants ainsi qu'avec la fréquence des interactions de vive voix avec ceux-ci. Le modèle final indique également que la perception du soutien des enseignants serait fortement et positivement liée à la valeur que les élèves attribuent à leur travail. Dans ce dernier modèle, l'effet de la fréquence des interactions de vive voix apparaît non significatif et l'effet de la fréquence des contacts est réduit en comparaison avec le modèle précédent. 
Concernant le sentiment d'efficacité personnelle relative au travail à domicile, nos analyses indiquent que cette variable est positivement liée à la fréquence des contacts avec les enseignants, mais négativement liée à la fréquence du travail à domicile. Le modèle final indique que la perception du soutien des enseignants serait également positivement liée au sentiment d'efficacité personnelle des élèves. Dans ce dernier modèle, l'effet de la fréquence des contacts avec les enseignants est à nouveau réduit.

Enfin, en ce qui concerne l'engagement comportemental des élèves, les résultats de nos analyses indiquent une forte relation positive avec la fréquence des contacts avec les enseignants. Cette relation serait plus importante que pour la valeur et le sentiment d'efficacité personnelle. La perception du soutien des enseignants serait également positivement liée à l'engagement, mais dans une moindre mesure que pour la valeur et le sentiment d'efficacité personnelle.

\section{Tableau 3}

Régressions linéaires multiples des variables de contrôle, de la fréquence de la continuité du travail scolaire et du lien social et du soutien perçu des enseignants sur la valeur de la tâche, le sentiment d'efficacité personnelle et l'engagement comportemental.

\begin{tabular}{|c|c|c|c|c|c|c|}
\hline \multirow[b]{2}{*}{ Origine } & \multirow{2}{*}{$\begin{array}{l}\text { Valeur } \\
1,81^{* * *}\end{array}$} & \multicolumn{2}{|c|}{ SEP } & \multicolumn{3}{|c|}{ Engagement } \\
\hline & & $1,06^{* * *}$ & $1,91^{* * *}$ & $1,39 * * *$ & $2,49^{* * *}$ & $2,10 * * *$ \\
\hline \multicolumn{7}{|c|}{ Variables contrôle } \\
\hline Genre & $-.08^{* * *}$ & $-.07^{* * *}$ & $.09 * * *$ & $.10^{* * *}$ & $-.08 * * *$ & $-.07 * * *$ \\
\hline \multicolumn{7}{|l|}{ Redoublement } \\
\hline SES & & & $.13^{* * *}$ & $.14^{* * *}$ & & \\
\hline \multicolumn{7}{|c|}{ Pratiques des enseignants } \\
\hline $\begin{array}{l}\text { Fréquence travail à } \\
\text { domicile }\end{array}$ & & & $-.07 * *$ & $-.09 * * *$ & & \\
\hline $\begin{array}{l}\text { Fréquence contacts } \\
\text { enseignants }\end{array}$ & $.23^{* * *}$ & $.10^{* * *}$ & $.21^{* * *}$ & $.12^{* * *}$ & $.30 * * *$ & $.21^{* * *}$ \\
\hline $\begin{array}{l}\text { Fréquences interactions } \\
\text { de vive voix avec les } \\
\text { enseignants }\end{array}$ & $.06^{* *}$ & Ns & & & & \\
\hline Soutien perçu & & $.46^{* * *}$ & & $.32 * * *$ & & $.27 * * *$ \\
\hline $\begin{array}{l}\text { Part de variance } \\
\text { expliquée }\end{array}$ & $8 \%$ & $26 \%$ & $6 \%$ & $15 \%$ & $10 \%$ & $16 \%$ \\
\hline
\end{tabular}

Note. Les coefficients de régression présentés sont standardisés.

${ }^{* * *} \mathrm{p}<.001 ;{ }^{* *} \mathrm{p}<.01 ;{ }^{*} \mathrm{p}<.05$ 


\section{Résultats}

Nos analyses soulignent tout d'abord l'importance d'avoir fréquemment des contacts avec les enseignants pour tous les aspects de la motivation et principalement pour l'engagement comportemental. Cependant, seule une courte majorité d'élèves rapportent avoir eu régulièrement des contacts avec les enseignants et plusieurs répondants font part dans les commentaires de leur souhait d'être davantage en contact. Plusieurs élèves rapportent cependant aussi de grandes variations dans ce qui a été mis en œuvre par leurs différents enseignants.

En ce qui concerne les interactions de vive voix avec les enseignants, notamment via des appels vidéo, on observe que seule une minorité a pu bénéficier de ce type de contacts. D'après les analyses multivariées, le fait d'avoir fréquemment des interactions de vive voix avec les enseignants n'aurait une plus-value que pour la valeur que l'élève attribue à son travail. Plus les élèves ont l'opportunité d'interagir oralement avec leurs enseignants, plus ils jugent leur travail scolaire comme étant intéressant, utile ou important. Parmi les commentaires libres, plusieurs indiquent avoir apprécié les visioconférences (les lives ou appels vidéo, selon les termes employés) et souhaiteraient que cette modalité soit davantage mise en œuvre en pareille situation (31 répondants). Ceux qui ont expérimenté les visioconférences pointent cependant certaines difficultés rencontrées : sessions programmées au même moment par différents enseignants, problèmes de connexion, etc.

Concernant la fréquence de travail transmis aux élèves, il apparaît que la majorité des élèves ont régulièrement reçu du travail à réaliser à la maison. Nos analyses indiquent que la fréquence d'envoi de travail, bien que positivement corrélée aux trois variables motivationnelles, ne semble pas contribuer à la motivation des élèves lorsque la fréquence des contacts est déjà prise en compte dans les analyses. De manière inattendue, nos résultats montrent même un effet négatif de la fréquence de travail sur le sentiment d'efficacité personnelle (alors que ces deux variables sont positivement corrélées). Nous avons donc testé un effet d'interaction entre la fréquence de travail et la fréquence de contacts dans un nouveau modèle, en centrant les variables indépendantes. Ces analyses complémentaires montrent que le facteur d'interaction est significatif $(B=0.057, \mathrm{p}=.005)$. Ces données semblent donc indiquer que l'effet négatif de la fréquence du travail à domicile sur le sentiment d'efficacité personnelle des élèves serait principalement présent chez les élèves qui rapportent une faible fréquence de contact avec les enseignants et ne concernerait pas les élèves rapportant avoir beaucoup de contacts avec ceux-ci. Les élèves qui reçoivent beaucoup de travail à réaliser, mais qui n'auraient que peu de contacts avec les enseignants se sentiraient donc moins capables de réaliser le travail attendu de leur part.

Certains professeurs nous ont littéralement submergé de travail chaque jour de la semaine, weekend et jours fériés compris. Ils ne se sont pas concertés et par moment, nous recevions des montagnes de devoirs à faire en même temps. [...] Notre professeur de néerlandais nous donnait tellement de matière que très souvent, je pleurais en recevant mes leçons et j'avais l'impression que je n'y arriverais jamais. [...].

[...] Les profs envoient trop de travail sans se préoccuper de comment on va. Au plus il y a de travail, au moins j'ai envie de travailler [...]. 
Parmi les 1717 répondants ayant laissé un commentaire à la question ouverte, 117 ont abordé la question de la fréquence ou, de manière plus large, du rythme et de la charge du travail à domicile, parmi lesquels 70 répondants ont indiqué recevoir trop de travail, sans forcément distinguer, à quelques exceptions près, si cela était dû à la fréquence ou à la nature des travaux demandés (difficulté ou longueur). Plusieurs indiquent également que cette fréquence pouvait fortement varier d'un moment à l'autre, ce côté imprévisible rendant l'organisation du travail et la planification d'activités difficiles. Certains répondants ont également indiqué qu'il était arrivé que beaucoup de travail leur soit transmis en même temps (ou pour la même date de remise) pour les différents cours. Dans la même idée, plusieurs répondants ont évoqué un manque de prise en compte de la part des enseignants du travail à fournir pour les autres cours ou un manque de concertation entre ceux-ci. Enfin, les élèves ont mentionné des délais trop courts pour rendre les travaux, ce qui pouvait générer du stress. À l'inverse, certains ont fait état de délais trop longs ou d'absence de délais pour remettre les travaux, ce qui ne les encourageait pas à les réaliser.

En lien avec la question du travail à réaliser à domicile, plusieurs répondants (35) ont évoqué l'importance d'un retour ou d'une correction alors que cette thématique n'était pas abordée dans le questionnaire. Cette absence de retour aurait selon les commentaires des élèves un impact négatif sur leur motivation.

\section{Certains professeurs ont beaucoup été présents pour moi et les camarades et ont vraiment cherché à ce que l'école à distance se passe bien. D'autres [...] nous expliquaient qu'ils avaient la "flemme " de corriger nos travaux puisque de toute façon ils étaient formatifs et pas certificatifs, pas très motivant pour travailler [...].}

Nos analyses mettent également en évidence l'importance du soutien perçu de la part des enseignants pour les différentes variables motivationnelles, tout particulièrement pour la valeur perçue, dont le soutien perçu explique à lui seul $18 \%$ de part de variance. Les élèves qui perçoivent que leurs enseignants s'intéressent à eux et sont disponibles pour les soutenir dans leurs apprentissages trouvent davantage que le travail à domicile est intéressant, utile ou important, se sentent davantage capables de réaliser le travail qui leur est demandé et s'y impliquent également davantage. Pour les trois variables motivationnelles, l'introduction du soutien perçu dans les modèles de régression a provoqué une diminution de l'effet de la fréquence des contacts, laissant penser que cette variable pourrait jouer un rôle médiateur (partiel) entre la fréquence des contacts et la motivation des élèves. Dans les commentaires laissés, de nombreux élèves mentionnent avoir le sentiment que les enseignants ne se soucient pas suffisamment de leur vécu, ce qui fait écho au faible taux d'élèves rapportant que leurs enseignants s'intéressent régulièrement à ce qu'ils vivent. Dans cette thématique du soutien, plusieurs élèves indiquent souhaiter un meilleur suivi de la part des enseignants. Plus spécifiquement, ils sont plusieurs à mentionner que les enseignants ne répondaient pas (rapidement) aux messages et questions envoyés, ce qui semblait affecter leur perception globale du soutien fourni par les enseignants.

Enfin, parmi les commentaires liés à la thématique de la motivation, plusieurs élèves font état d'une dégradation de celle-ci au fur et à mesure que le temps passait (17). Si plusieurs élèves ont mentionné ne pas avoir envie de travailler, d'autres ont exprimé le fait de ne pas arriver à travailler ou à se concentrer (36), certains attribuant cela à une difficulté à s'organiser et à instaurer pour soi-même un rythme et un cadre de travail adéquats. 


\section{Limites}

La présente étude comporte cependant plusieurs limites. Tout d'abord, notre échantillon ne semble pas représentatif de la population de référence, ce qui est problématique au regard de notre premier objectif visant à documenter ce qui avait été mis en place par les enseignants en Fédération WallonieBruxelles. Ensuite, les items utilisés sont adaptés d'études précédentes, mais ne sont peut-être pas les plus adéquats en ce contexte inédit. Les commentaires des élèves ont fait émerger des thématiques habituellement moins prises en compte (délais des travaux, capacité à s'auto-organiser, etc.) et ont mis en évidence les termes employés par les élèves pour les nouvelles modalités, ce qui permettra d'affiner nos outils à l'avenir. Enfin, étant donné le caractère transversal de cette étude, on ne peut réellement tirer de conclusions quant au sens de la causalité entre les variables, notamment entre perception de soutien et motivation des élèves. Il se peut en effet que les élèves davantage motivés perçoivent plus positivement ce que mettent en place leurs enseignants ou encore que les enseignants soient davantage soutenants avec les élèves qui sont motivés.

\section{Implications pratiques}

Plusieurs implications pratiques peuvent être tirées de cette étude afin de soutenir la motivation des élèves ne pouvant plus se rendre à l'école dans un contexte de pandémie. Ces recommandations peuvent aussi avoir leur pertinence en situation d'enseignement mixte ou hybride.

Tout d'abord, établir des contacts avec les élèves apparaît comme une nécessité pour soutenir leur motivation. Pris par le temps, des enseignants pourraient choisir de n'envoyer que du travail à réaliser (par mail ou via un autre canal) sans prendre vraiment contact, se disant que cela est mieux que rien. Nos résultats indiquent que cette croyance est erronée et que les élèves qui reçoivent énormément de travail sans être en lien et suivis par les enseignants ne se sentent parfois plus capables de réaliser le travail demandé et risquent de se décourager. Parmi les commentaires libres, plusieurs élèves attirent l'attention sur l'importance d'un délai adapté pour la remise de ces travaux (ni trop court, ni trop long). De même, les élèves semblent particulièrement sensibles à la réception d'un retour sur le travail réalisé et, idéalement, d'une correction individualisée (plutôt que la simple transmission d'un correctif global). Concernant ce besoin de feedback, une piste suggérée par plusieurs élèves semble intéressante à mentionner. Ceux-ci rapportent en effet apprécier particulièrement la réalisation de questionnaires en ligne, qui permet une rétroaction immédiate.

Ensuite, la possibilité d'échanger oralement avec l'enseignant apparaît également comme importante. Mettre en place des cours en visioconférence semble notamment une piste intéressante, à condition d'être attentif à différents enjeux : les répartir de manière adéquate au sein de la journée et de la semaine, limiter leur nombre ou leur durée en prenant en compte les capacités attentionnelles des élèves face à un écran, veiller à l'accès au contenu pour ceux qui rencontreraient des difficultés techniques, instaurer un cadre et une régularité permettant un climat de travail favorable, etc. L'envoi de vidéos pourrait également répondre au besoin exprimé par les élèves d'avoir des explications orales concernant la matière et les consignes du travail à réaliser. À nouveau, ces vidéos devraient éviter certains écueils : pas trop longues, rythme adapté, dynamisme et insertion d'illustrations, etc. 
La disponibilité des enseignants et le fait de répondre (dans un délai raisonnable) aux questions qui leur sont posées (par mail ou via les plateformes) apparaissent comme des éléments importants aux yeux des élèves pour soutenir leur motivation. On imagine aisément que gérer les mails individuels représente une surcharge importante pour les enseignants, qui doivent déjà adapter fortement leurs pratiques. Afin que les élèves se sentent " suivis » et non " abandonnés ", les enseignants pourraient instaurer un cadre en fixant une plage horaire sur la semaine durant laquelle les élèves peuvent s'attendre à recevoir une réponse. Des forums en ligne pourraient également être mis en place, afin que l'ensemble des élèves puissent bénéficier des échanges, mais également y contribuer en répondant aux questions ou demandes d'explications de leurs pairs.

Devant la difficulté de se mettre au travail et de se concentrer, rapportée notamment par certains élèves se disant pourtant motivés, il semble essentiel de contribuer à instaurer un cadre clair et des routines pour les élèves devant rester à domicile. Avant tout, les modalités choisies par l'école et les enseignants pour communiquer et transmettre le travail scolaire ne devraient pas constituer une surcharge cognitive pour les élèves. Réduire le nombre d'outils informatiques et de plateformes utilisés pour une même classe apparaît nécessaire au vu des propos rapportés par les élèves. La demande de travail à domicile devrait également être répartie de manière adéquate dans le temps (ainsi que les délais de ces travaux), ce qui implique une concertation entre enseignants ou, éventuellement, la mise en place d'un journal de classe en ligne. Idéalement, dans l'idée de fournir un cadre clair et structurant, ces demandes de travaux ne devraient pas arriver de manière inattendue ni en dehors des heures « scolaires ». Rappelons toutefois que « cadre » ne signifie pas « contrôle » et que, plus encore en contexte de pandémie, il est important de laisser de l'autonomie aux élèves. Ils sont notamment nombreux à rapporter avoir profité de cette période pour réaliser d'autres apprentissages «non scolaires » ou se consacrer à des passions, ce qui contribuait selon eux à leur bien-être dans ce contexte particulier. Pour revenir au travail scolaire, former explicitement les élèves à l'auto-régulation et l'auto-organisation pourrait également se révéler une initiative utile (en plus de former aux outils technologiques), surtout pour les plus jeunes.

Toutes ces recommandations impliquent que les enseignants soient informés, formés et soutenus afin d'adapter leurs pratiques en contexte de pandémie. En effet, la distance physique rend plus difficile la rétroaction par rapport à ce qu'ils mettent en place auprès des élèves, mais également les échanges de pratiques entre collègues. Les directions d'école ont sans doute là un rôle majeur à jouer, en plus de la coordination autour des outils technologiques.

Enfin, l'enseignement à distance en contexte de pandémie représente un réel changement de paradigme (UNESCO, 2020). Pour la période concernée, les propos rapportés par les élèves semblent indiquer que l'école a, pour beaucoup, continué à domicile sous d'anciennes formes. Certains élèves disent faire leurs " devoirs » à domicile ou encore recevoir chaque jour les sept ou huit travaux des cours prévus dans un horaire-type. Afin d'accompagner le nécessaire changement de mentalité auprès des différents acteurs de l'éducation, le choix de nouveaux termes spécifiques pour désigner les différents types d'activités d'apprentissage en contexte de pandémie pourrait davantage marquer la rupture et ainsi favoriser l'adaptation des pratiques des enseignants, au bénéfice de la motivation des élèves. 


\section{Références}

Bandura, A. (1980). L'apprentissage social. Mardaga.

Deci, E. L. et Ryan, R. M. (2000). The 'what' and 'why' of goal pursuits: Human needs and the self-determination of behavior. Psychological Inquiry, 11, 227-268. https://doi.org/10.1207/S15327965PLI1104 01

Di Pietro, G., Biagi, F., Costa, P., Karpiński Z. et Mazza, J. (2020). The likely impact of COVID-19 on education: Reflections based on the existing literature and international datasets, EUR 30275 EN, Publications Office of the European Union, https://doi.org/10.2760/126686

Eccles, J. S. et Wigfield, A. (1995). In the mind of the actor: The structure of adolescents' academic achievement relatedbeliefs and self-perceptions. Personality and Social Psychology Bulletin, 21(3), 215-225. https://doi.org/10.1177/0146167295213003

Federici, R. A. et Skaalvik, E. M. (2014). Students' Perceptions of Emotional and Instrumental Teacher Support: Relations with Motivational and Emotional Responses. International Education Studies, 7(1), 21-36. https://doi.org/10.5539/ies.v7n1p21

Fredricks, J. A., Blumenfeld, P. C. et Paris, A. H. (2004). School engagement: Potential of the concept, state of the evidence. Review of Educational Research, 74(1), 59-109. https://doi.org/10.3102/00346543074001059

Galand, B. et Hospel, V. (2011). État des lieux du risque de décrochage scolaire en Région Bruxelloise : Rapport de l'enquête menée par les cellules de veille. Rapport non publié, Université catholique de Louvain.

Galand, B. et Philippot, P. (2002). Style motivationnel des élèves du secondaire : développement d'un instrument de mesure et relations avec d'autres variables pédagogiques. Canadian Journal of Behavioural Science/Revue canadienne des sciences du comportement, 34(4), 261-275. https://doi.org/10.1037/h0087179

Hospel, V., Galand, B. et Janosz, M. (2016). Multidimensionality of behavioural engagement: Empirical support and implications. International Journal of Educational Research, 77, 37-49. https://doi.org/10.1016/j.ijer.2016.02.007

Olivier, E., Galand, B., Hospel, V. et Dellisse, S. (2020). Understanding behavioural engagement and achievement: The roles of teaching practices and student sense of competence and task value. British Journal of Educational Psychology, 90(4), 887-909. https://doi.org/10.1111/bjep.12342

UNESCO (2020). Stratégies d'apprentissage à distance face à la fermeture des écoles due au COVID-19. https://unesdoc.unesco.org/ark:/48223/pf0000373305 fre

\section{Pour citer cet article}

Baudoin, N., Dellisse, S., Lafontaine, D. (2020). Soutien des enseignants et motivation des élèves durant la pandémie de COVID-19. Formation et profession, 28 (4 hors-série), 1-13. http://dx.doi.org/10.18162/fp.2020.688 